\title{
Supplementary Materials for "On the Relationship between Teleconnections and Taiwan's Streamflow: Evidence of Climate Regime Shift and Implications for Seasonal Forecasting" by Chia-Jeng Chen and Tsung-Yu Lee
}

\section{Caveats of using the CRS detection method:}

As already indicated by the manuscript, there are several classes of approaches available for the detection of regime shifts, such as parametric methods (e.g., the classical $t$-test), non-parametric methods (e.g., the Mann-Whitney-Pettitt test), regression-based methods, cumulative sum methods, and sequential methods. Rodionov (2005) pointed out the pros and cons of some common approaches as well as his sequential method (Rodionov, 2004). The pros of his method are the automatic, early detection of a regime shift and the ability to monitor a possibility of a regime shift in real time. He has shown that his method can outperform Lanzante's method (another robust, non-parametric procedure developed by Lanzante, 1996). Therefore, since developed, Rodionov's method has been used in many studies in climate sciences. Nevertheless, the cons of his method are the requirement of some experimentation on the two parameters used (i.e., the cut-off length / and probability level $p$ ) and inability to account for a gradual regime shift and data with obvious autocorrelation (or red noise, but this issue was later ameliorated by a prewhitening procedure introduced by him, Rodionov, 2006). According to Rodionov (2004), while the probability level $p$ is known to determine the critical value of $t$ (the Student's $t$-distribution), "the cut-off length I determines the minimum length of the regimes, for which the magnitude of the shifts remains intact." It has been tested that, the larger / is set, the fewer change points can be identified. By contrast, a smaller / does not necessarily lead to more change points since only those significant change points can be identified based on the $t$-test. In other words, if there is no strong regime shift in the data series, the method with some variations in $p$ and / simply cannot identify any change point (Rodionov, 2015 and verified by us too). In any case, we should still use Rodionov's method with caution since the CRS detection method is purely statistical, and the CRS results could be meaningless without solid evidence from related research. 


\section{Supplementary Figures:}

$\rightarrow-P J \_J A S-P J \_J J A$

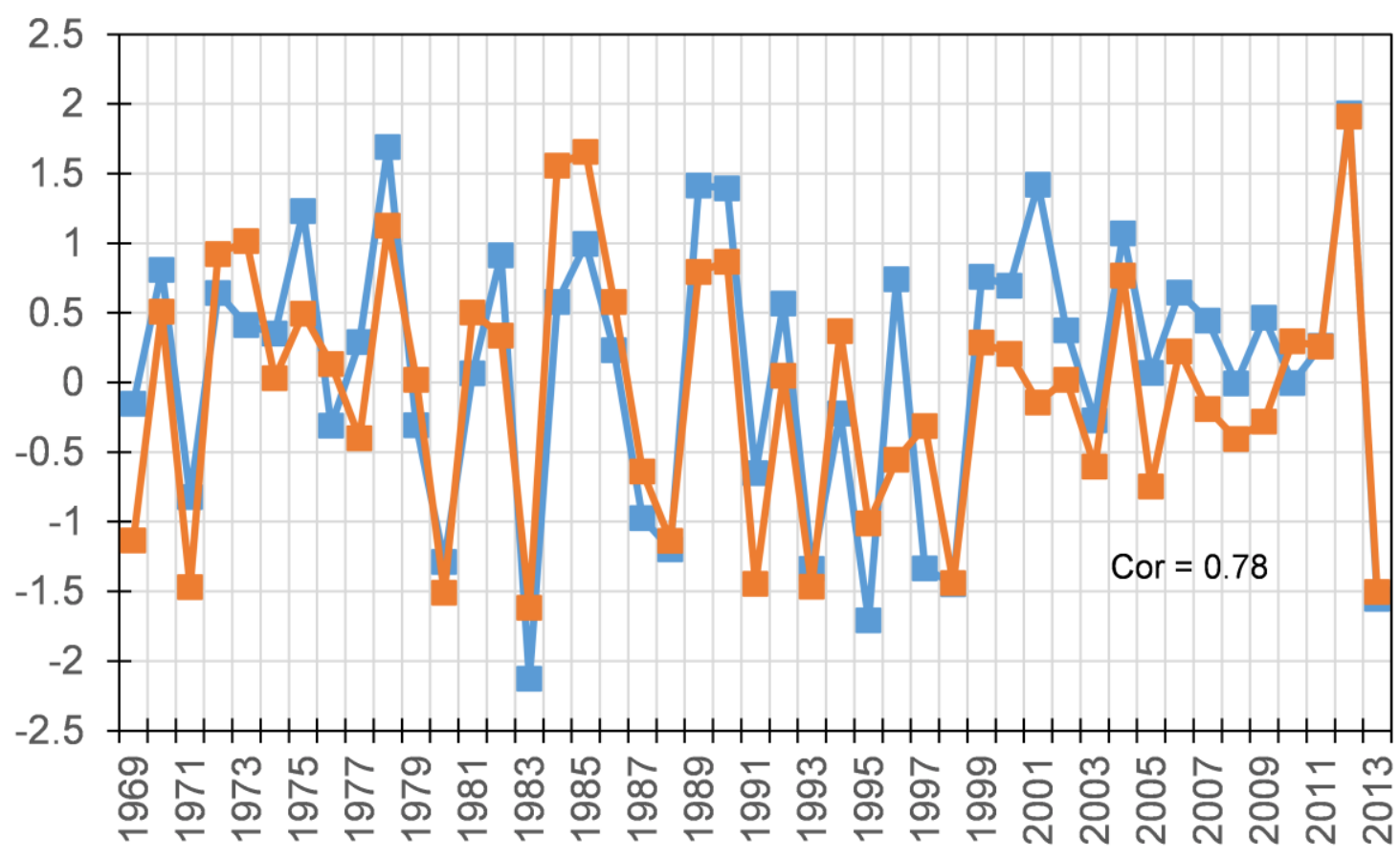

Figure S1. Comparison between the PJ index in JAS and that in JJA.

$\rightarrow-$ Critical value of field sig. $(p=0.05) \rightarrow-$ Observed value

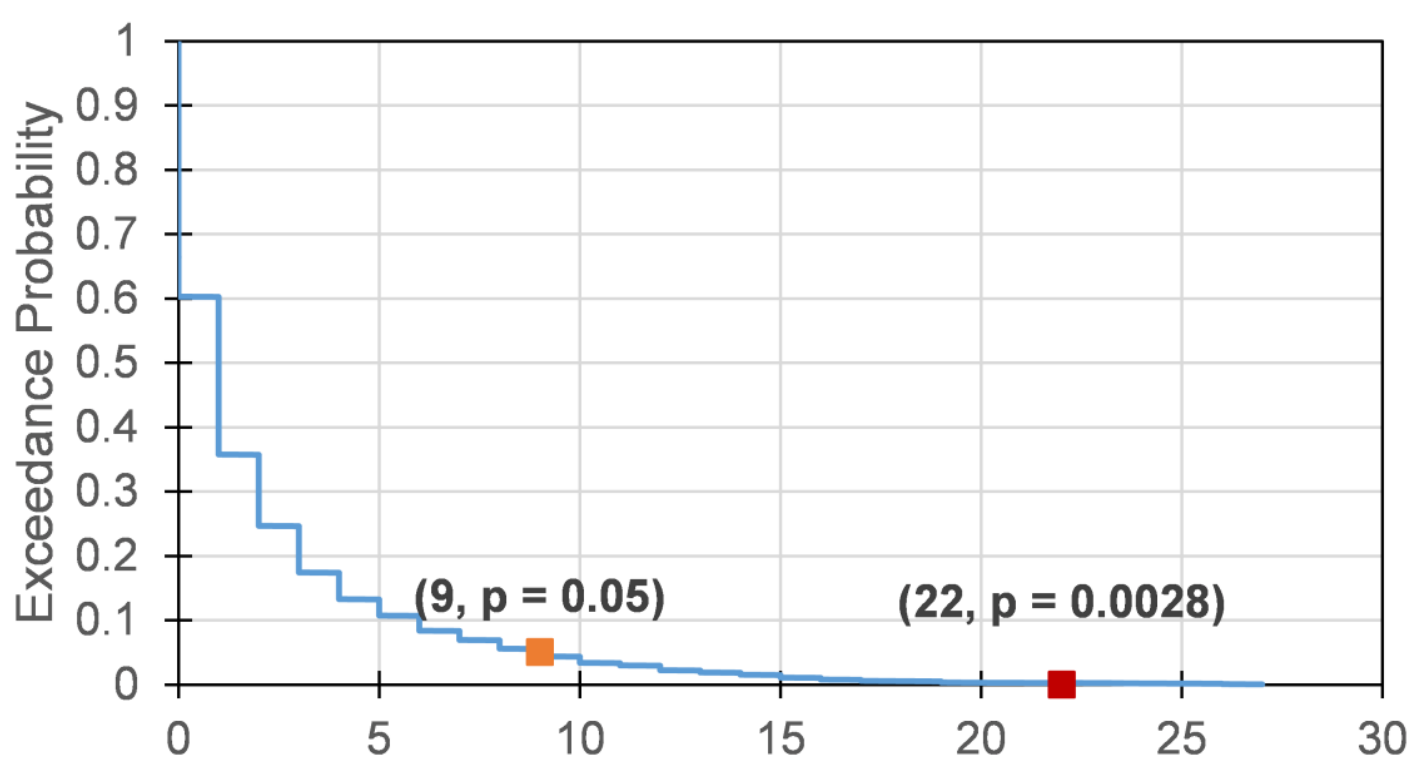

Figure S2. Empirical null distribution of field significance test. The abscissa represents the number of catchments with correlations between streamflow and lagged QBO significant at the 95\% level $(p=0.05)$ in 2000 Monte Carlo trials. 

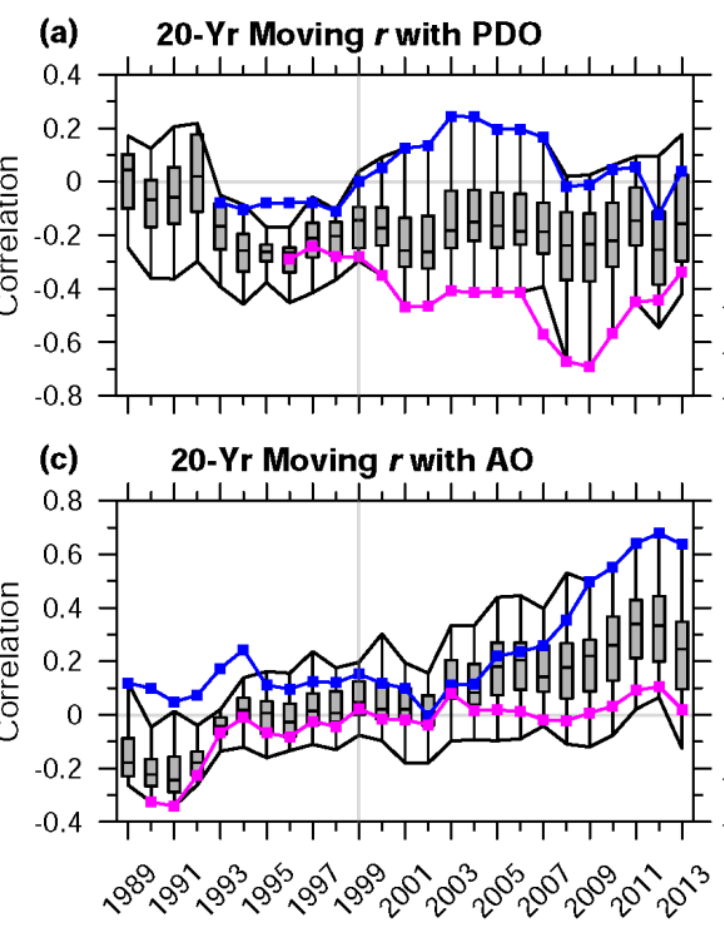

(b) 20-Yr Moving $r$ with EPNP

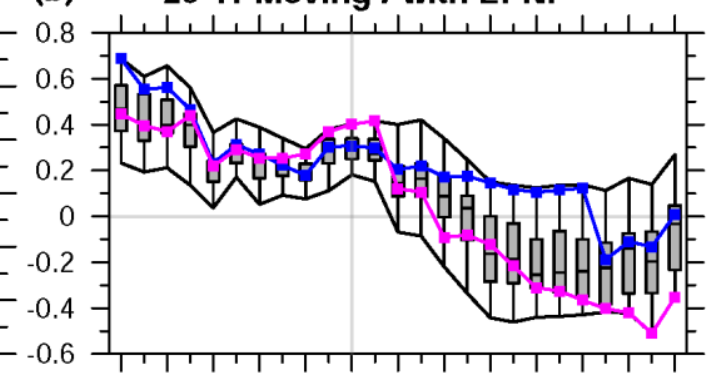

(d)

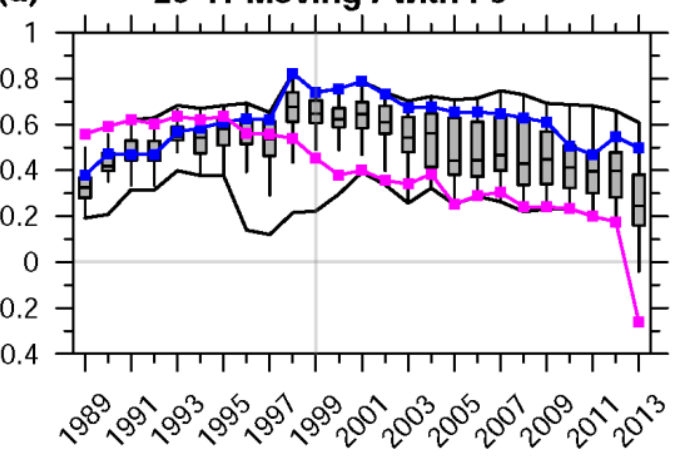

Figure S3. Selected moving-window correlation results. Each boxplot encapsulates correlation values derived from 28 upstream catchments (JAS runoff vs. specific climate index). The year label indicates the end of a moving window (e.g., 1989 indicates the moving window is taken from 1970 to 1989).

Blue (magenta) time series denotes the highest (lowest) moving-window correlations over the temporal horizon, and can go outside the box plot range (i.e., deemed an outlier) since the IQR with a factor of 1.5 is used to determine a rather diminished range. 

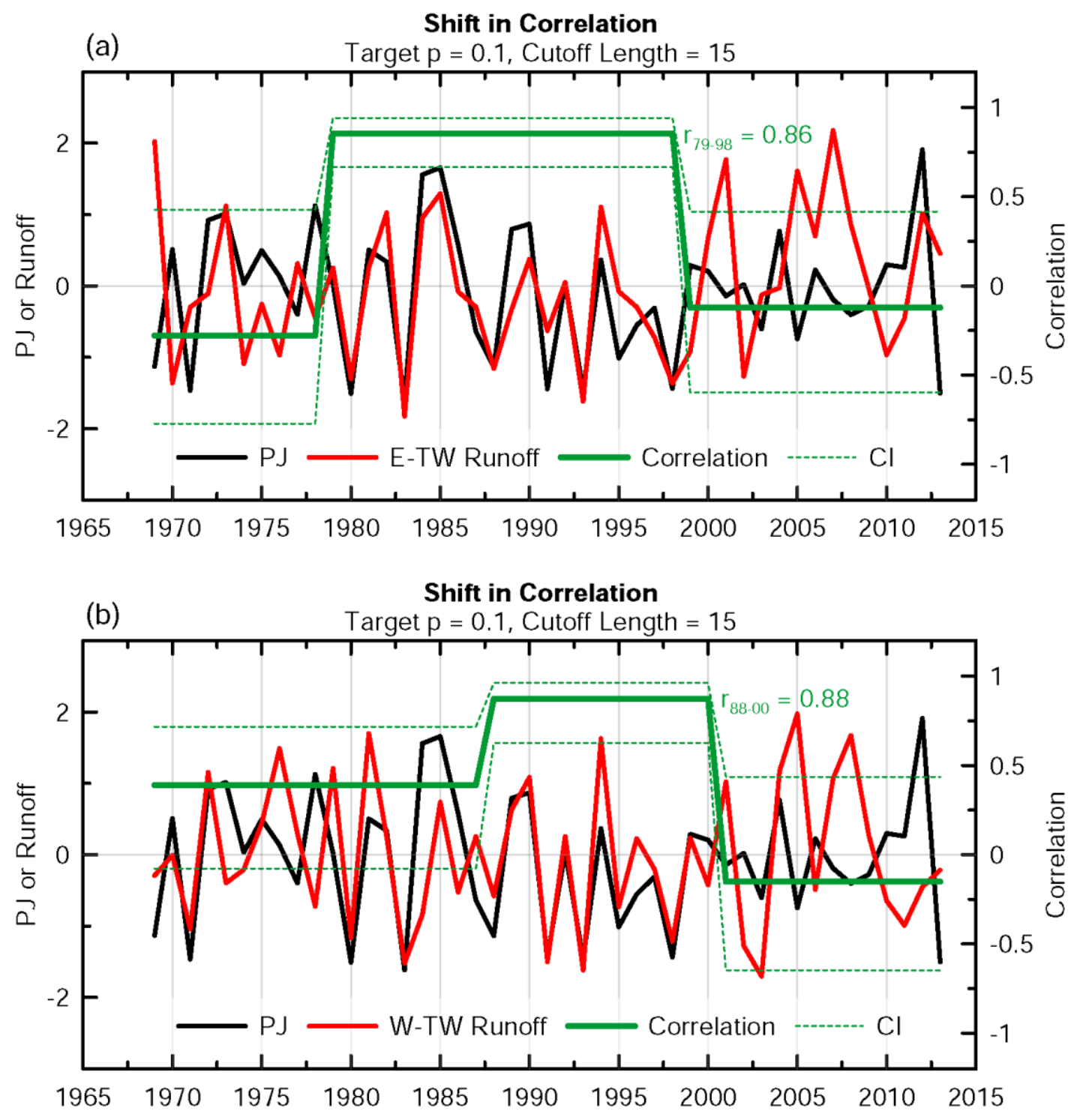

Figure S4. Shifts in the correlation between (top: East; bottom: West) Taiwan runoff and the PJ index.

\section{References}

Lanzante, J.R., 1996. Resistant, robust and non-parametric techniques for the analysis of climate data: Theory and examples, including applications to historical radiosonde station data. International Journal of Climatology, 16(11), pp.11971226.

Rodionov, S.N., 2005. A brief overview of the regime shift detection methods. Large-Scale Disturbances (Regime Shifts) and Recovery in Aquatic Ecosystems: Challenges for Management Toward Sustainability, pp.17-24. 
Rodionov, S.N., 2006. Use of prewhitening in climate regime shift detection. Geophysical Research Letters, 33(12). 